\title{
Comparative Study of 2-DOF Micromirrors for Precision Light Manipulation
}

\author{
Johanna I. Young and Andrei M. Shkel \\ Microsystems Laboratory, Department of Mechanical and Aerospace Engineering, \\ University of California, Irvine, CA, USA
}

\begin{abstract}
Many industry experts predict that the future of fiber optic telecommunications depends on the development of all-optical components for switching of photonic signals from fiber to fiber throughout the networks. MEMS is a promising technology for providing all-optical switching at high speeds with significant cost reductions. This paper reports on the the analysis of two designs for 2-DOF electrostatically actuated MEMS micromirrors for precision controllable large optical switching arrays. The behavior of the micromirror designs is predicted by coupled-field electrostatic and modal analysis using a finite element analysis (FEA) multi-physics modeling software. The analysis indicates that the commonly used gimbal type mirror design experiences electrostatic interference and would therefore be difficult to precisely control for 2-DOF motion. We propose a new design approach which preserves 2-DOF actuation while minimizing electrostatic interference between the drive electrodes and the mirror. Instead of using two torsional axes, we use one actuator which combines torsional and flexural DOFs. A comparative analysis of the conventional gimbal design and the one proposed in this paper is performed.
\end{abstract}

Keywords: Optical MEMS, Micromirror, and Coupled-field Modeling

\section{INTRODUCTION}

The growing demand on telecommunication networks for large bandwidth at high speeds is an issue which has attracted much investment of time and research funds by companies attempting to market products which will make "all-optical" high-speed networking a reality. The light waves that carry data and travel through optic fibers must be switched to other fibers throughout the networks in order to reach their destination. The current switching technology used to accomplish this processes data electronically which means the photonic signals must be converted to electrical signals, then switched and converted back to light waves. These Optical-Electrical-Optical (OEO) conversions slow down the rate of data transmission and are very costly. With the advancement in recent years of DWDM (Dense Wavelength Division Multiplexing), several wavelengths can be sent through a single fiber and the electrical switching cores are not able to process the high bandwidth at a comparable speed to which it travels through the fibers, causing "data traffic jams." Networks with all-optical switches will be able to switch light waves extremely fast because no electrical conversions are needed. MEMS (Micro-Electro-Mechanical Systems) micromirrors used for optical switching applications offer fast switching times in millisecond range and can be built in scalable arrays to reflect transmitted light from fibers to the receiving fibers. MEMS micromirror optical switches will be faster, smaller, and less expensive than the current switches, while also being essentially future-proof because they have no maximum bandwidth limit as electrical switches do. There are several design and control issues that must be further investigated in order to implement large-scale switching arrays in networks. One important issue is the precision control of micromirrors. Very slight errors in mirror deflection will cause the photonic signal to miss the intended receiving fiber. An accurate measurement of mirror positioning is needed for precision control with feedback. Current prototype micromirror array optical switches do not include advanced control and will therefore not deliver the precision necessary for large arrays. In this paper, we analyze two designs for a 2-DOF (Degree-of-Freedom) MEMS micromirror and their potential for delivering precision light manipulation for telecommunication applications. The first issue to be addressed is the mirror positioning accuracy required for fiber to mirror coupling. Then two designs of a 2-DOF micromirror are analyzed and compared based on which design offers minimal electrostatic interference and can thus be more readily controlled to deliver the required precision.

Further author information: (Send correspondence to J.I.Y.)

J.I.Y.: E-mail: jiyoung@uci.edu

A.M.S.: E-mail: ashkel@uci.edu

Website: http://mems.eng.uci.edu 


\section{ESTIMATION OF PRECISION REQUIREMENTS}

Networks will require different sizes of switching arrays for different applications and the necessary precision of the angular positioning of the mirrors is dependent on the array size. The smaller array dimensions of $4 \times 4,8 \times 8$, and up to $32 \times 32$ will not require the same precision control as larger arrays of up to 1,152x1,152 mirrors. The smaller arrays usually consist of 1-DOF mirrors which flap up or down through 90 degrees of rotation to either reflect a light beam or let light beams pass over or under them ${ }^{1,2}, 3$ The mirrors can be actuated electrostatically or magnetically and rest against a hard stop in their "on/off" position created for example by a polysilicon layer in the surface micromachining fabrication process ${ }^{3,2}$. Minor errors in mask alignment during the fabrication process might offset these hard stops slightly, causing a small change in the angle at which light will be reflected. For smaller arrays,

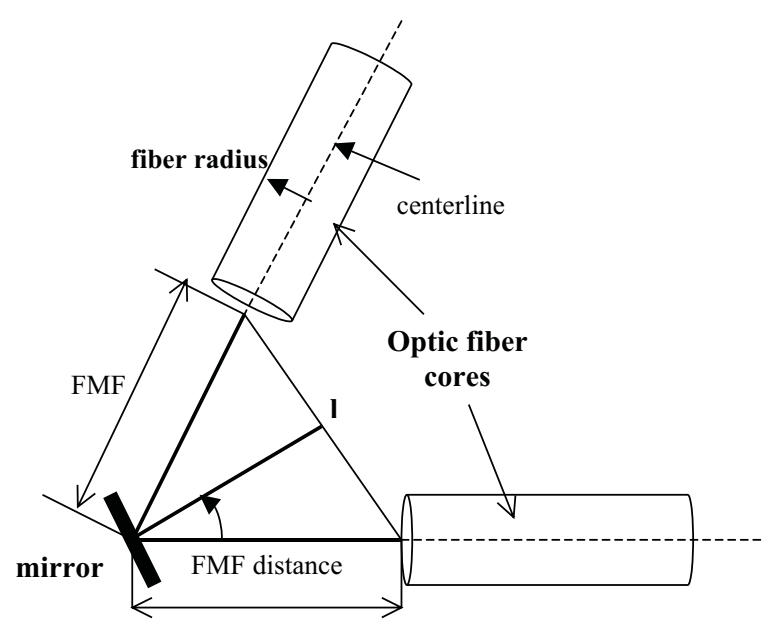

(a)

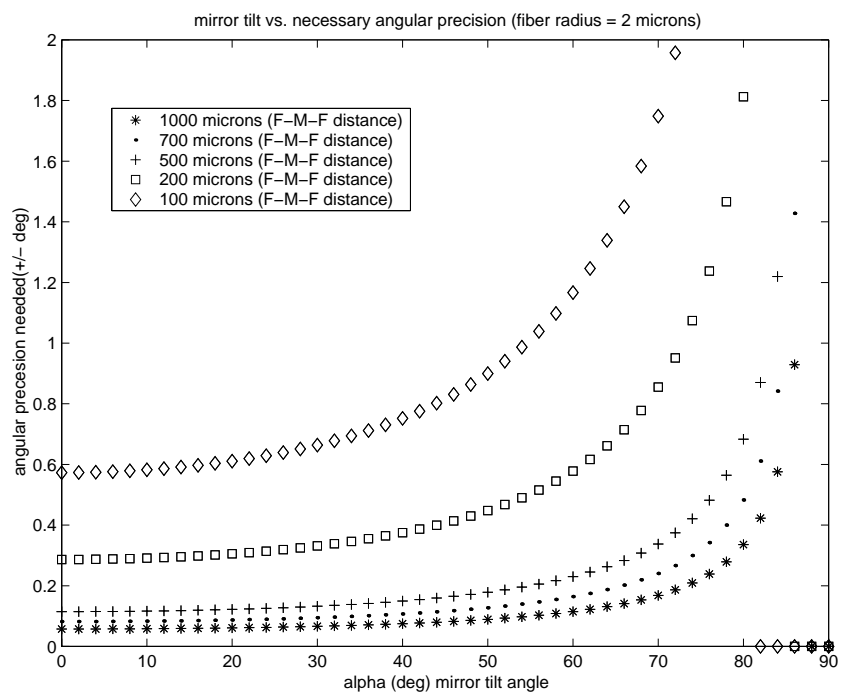

(b)

Figure 1. a.) Two-dimensional fiber-mirror-fiber schematic used for calculation of necessary precision. b.) Necessary precision vs. mirror tilt angle for varying distances between fibers and mirror.

this small error will likely not cause problems because the receiving fibers will be in close enough range to still receive the signal. But for larger arrays, this two-stage (on/off) open-loop control will not be sufficient to fully transmit signals to fibers which are at distances of hundreds of mirrors away. The slight angular offset error will increase over distance and the light wave will miss the targeted fiber port. Larger arrays must have 2 or 3-DOF and will require advanced control with feedback in order to meet the strict positioning requirements for complete signal transmission from mirrors to fibers at increasingly greater distances corresponding to larger arrays. We estimated precision requirements using a two-dimensional schematic (see Fig. 1(a)). The diameter of a single-mode fiber core is approximately 2 to 8 micrometers, and the total diameter of a coated fiber is about 100 micrometers. Using the 2-D schematic, for a given angle of mirror tilt, $\alpha$, the range for reception of the transmitted signal is the distance from the centerline (in the positive or negative direction) of the receiving fiber to the edge of the fiber core. Thus, the radius of the fiber is the parameter that determines the range of allowable mirror tilt error. It is assumed that the light wave is ideally collimated with a Gaussian profile. This model does not account for the minimum angle of incidence at which the light wave must enter the fiber in order for total internal reflection of the light wave to occur, and thus the actual precision requirements will be stricter than those calculated with the simplified 2-D geometry. The mirror is assumed to only function in the range of $\alpha$ equal to 0 through 90 degrees. Using the trigonometry of the schematic (See Eqns. 1-4) plots were made to determine the angular precision necessary for given tilt angles at fixed fiber-mirror-fiber (FMF) distances (see Fig. 1(b)). From the Law of Cosines and for a given tilt angle, $\alpha$ : $l^{2}=d^{2}+d^{2}-2 d^{2} \cos (2 \alpha)$. In the following equations used for calculating the required angular precision, $P$, the parameter $r$ is the fiber core radius, $l$ is the distance between the optical axes of the source and receiving fiber, and 
$d$ is the fiber to mirror distance (FMF).

$$
\begin{gathered}
l=\sqrt{2 d^{2}(1-\cos (2 \alpha))}, \\
l_{\text {new }}=l+r, \\
\alpha_{\text {new }}=\frac{1}{2} \arccos \left(1-\frac{l_{\text {new }}^{2}}{2 d^{2}}\right), \\
P= \pm\left(\alpha_{\text {new }}-\alpha\right) .
\end{gathered}
$$

Our calculations illustrate that for a distance of 500 microns, that is approximately a $5 \times 5$ mirror array, and a fiber core diameter of 4 microns, 0.1 degree precision of micromirror positioning is required (see Fig. 1(b)). Thus, with open-loop control the precision required for 1,152x1,152 mirror arrays, as called for in telecommunication applications, would be extremely difficult to achieve.

\section{COMPARATIVE STUDY OF 2-DOF MIRROR DESIGNS}

There are several factors which can contribute to the ability or inability of a micromirror design to be controllable to precise 2-DOF positions. In the previous section we studied the mirror positioning precision requirements and found that open-loop control would not be feasible for large arrays. In this section we consider two micromirror designs and their capability for providing 2-DOF precision positioning by performing electrostatic and modal analyses. We find that the commonly used gimbal mirror design experiences electrostatic interference among its four electrodes which presents problems in attaining 2-DOF motions and modes which would be beneficial for optical switching. We then propose a new design which preserves 2-DOF actuation and offers desirable modes while minimizing electrostatic interference by using only two electrodes. This new design will be more readily controllable to precise positions with feedback control because of the reduced electrostatic interference.

\subsection{Gimbal Mirror Design and Analysis}

One of the studied 2-DOF mirror designs consists of a rectangular mirror attached to a frame (See Fig. 2(a)). Both the mirror and the frame are free to rotate with respect to orthogonal axes. This design is similar to one explored by several authors ${ }^{4,} .5$ This device is actuated using four electrodes; two are placed under the mirror and another two under the frame. To verify sensitivity of the device and investigate effects of electrostatic cross-coupling we modeled



(a)



(b)

Figure 2. a.) Mirror-in-Frame (gimbal) design modeled in FEA software. b.) Location of voltage applications in FEA model and desired 2-DOF rotations.

this mirror in ANSYS software using the built-in electrostatic-structural coupled-field solver, ESSOLV. Three cases of actuation were modeled: (1) voltage is applied to only one mirror electrode, (2) voltage is applied only to one frame electrode, and (3) voltage is applied to both the mirror and the frame electrode, (See Fig. 2(b)). The results show that when both mirror and frame are actuated, electrostatic interference creates undesired coupling of rotations (See Fig. 3(a),(b), and (c)). When 50 Volts is applied to the mirror electrode (Case(1)), the mirror rotates with respect to its beam axis by an angle of $\alpha_{1}=0.1165$ degrees (See Fig. 3(a)). When 50 Volts is applied to both the frame and mirror electrode (Case(3)), the mirror rotates with respect to its beam axis by an angle of $\alpha_{3 \max }=0.0862$ degrees 




(a)

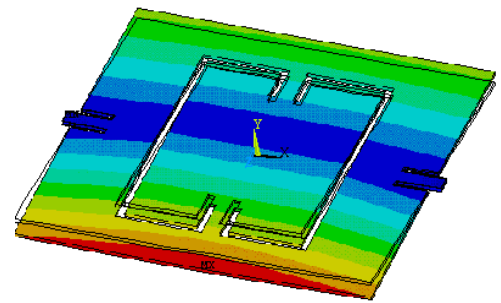

(b)

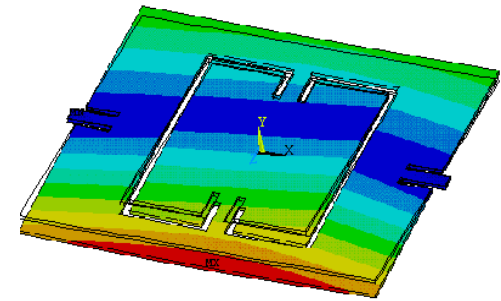

(c)

Figure 3. FEA electrostatic-structural coupling of gimbal mirror design. a.) Voltage applied only to one mirror electrode. Maximum vertical deflection of mirror edge $=0.1017 \mu \mathrm{m}$, rotation of mirror about mirror beam axis $=$ 0.117 degrees. b.) Voltage applied only to one electrode under frame. Maximum vertical deflection of frame $=0.5958$ $\mu \mathrm{m}$, rotation of frame about frame beam axis $=0.325$ degrees. c.) Voltage applied to one mirror electrode and one frame electrode. Maximum vertical deflection of frame $=0.6777 \mu \mathrm{m}$, rotation of frame about frame beam axis $=$ 0.370 degrees. Rotation of mirror about mirror beam axis $=0.086$ degrees .

(See Fig. 3(c)). The decrease in rotation angle of the mirror between Case(1) and Case(3) is due to the electrostatic interference from the frame electrode which has more influence in pulling the mirror to rotate with the frame then for the mirror to rotate orthogonally with respect to the frame as actuated by the mirror electrode. When the mirror is tilted in rotating within the frame, the electrostatic force between the mirror and mirror electrode is greater at the deflected edge near the activated frame electrode because electrostatic force increases non-linearly with decreasing gap distance. This difference in force along the mirror edge causes unsymmetric rotation of the mirror about it's beam axis in Case(3). The $\alpha_{3}$ measured from the edge of mirror opposite the activated frame electrode is less than the $\alpha_{3}$ measured from the mirror edge closest to the frame electrode, $\alpha_{3 \max }$. In the case when only the frame is actuated by 50 Volts (Case(2)), the frame rotates with respect to its beam axis by an angle of $\beta_{2}=0.3251$ degrees (See Fig. 3(b)). The rotation of the frame increases in Case(3) to $\beta_{3}=0.3698$ degrees. Ideally to obtain $2-\mathrm{DOF}$ the device should achieve commutative rotations, where Case(1) combined with Case(2) would produce the results of Case(3), but the results demonstrate the inability of the gimbal mirror design to achieve independent rotations of the mirror and frame about orthogonal axes. In order to obtain 2-DOF motion, the mirror and frame must rotate independently about their respective beam axes, but due to the electrostatic interference, the motions cannot be decoupled. In the previously cited reference ${ }^{4}$ the authors present a diagram based on experimental results of a similar gimbal mirror design plotting one axis rotation angle versus the other axis rotation angle which correlates with our findings of dependent motion.

It will be beneficial for optical switching applications to drive micromirrors into resonance in a desired modal shape because resonance requires lower voltages than needed for static deflections. A modal analysis performed on the gimbal mirror design also demonstrated coupling of rotations about the two orthogonal axes without achieving a mode in which the frame and mirror rotate independently about their respective beam axes. (See Table 1 and Fig. 4). These results along with the results from the electrostatic-structural analysis indicate this design will be difficult to control to achieve the required precision of large arrays of micromirrors.

Table 1. First three modes of gimbal mirror design as modeled in FEA software.

\begin{tabular}{|c|c|c|}
\hline Mode 1 & Mode 2 & Mode 3 \\
\hline $\begin{array}{c}\text { 64.508 } \mathrm{kHz} \\
\text { rotation about frame beam axis } \\
\text { (See Fig. 4(a)) }\end{array}$ & $\begin{array}{c}100.129 \mathrm{kHz} \\
\text { bowing of structure } \\
\text { (See Fig. 4(b)) }\end{array}$ & $\begin{array}{c}187.708 \mathrm{kHz} \\
\text { rotation about mirror beam axis } \\
\text { (See Fig. 4(c)) }\end{array}$ \\
\hline
\end{tabular}




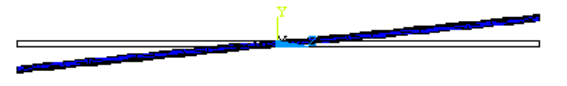

(a)

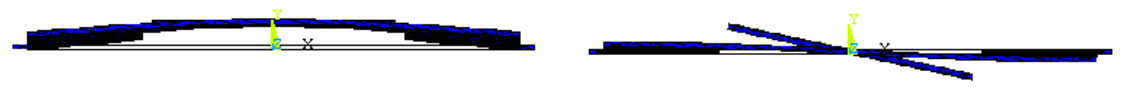

(b) (c)

Figure 4. a.) Mode 1. Side view of mirror and frame rotating about frame beam axis b.) Mode 2 . Front view of mirror and frame bowing out of plane. c.) Mode 3. Front view of mirror and frame rotating about mirror beam axis.



(a)

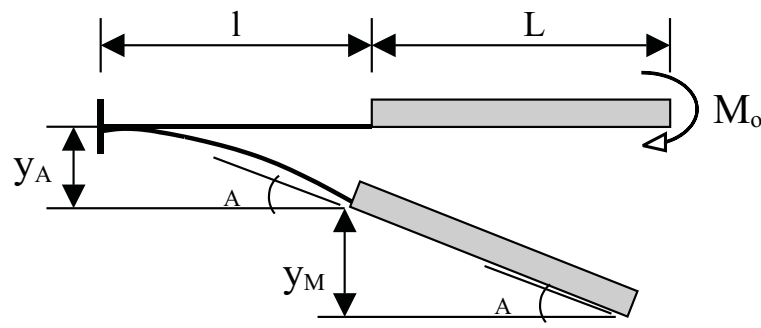

(b)

Figure 5. a.) Cantilevered beam mirror design with two rotation angles due to voltage bias and voltage difference on electrodes. b.) Beam and mirror static deflection due to applied moment.

\subsection{Cantilevered Beam Mirror Design and Analysis}

We propose a new simplified optical switch mirror design with advantages over the mirror-in-frame design while preserving $2-\mathrm{DOF}$ motion. ${ }^{6} \quad$ Electrostatic and modal analysis of the cantilevered beam mirror design exhibited less electrical interference than the gimbal mirror design and the desired 2-DOF motion was modeled. An added benefit of this design includes only two electrodes instead of four thus decreasing the complexity of on-chip wiring for independently addressed mirrors. The design consists of a mirror plate attached to the free end of an anchored cantilevered beam and suspended over the two electrodes. A voltage bias and a voltage difference over the electrodes controls orientation of the mirror (see Fig. 5(a)). When a bias voltage is applied to both electrodes, the mirror displaces vertically and is tilted by beam bending (see Fig. 6(a)). When a voltage difference exists the mirror is deflected vertically toward the electrodes and rotates about the beam axis toward the electrode with the greater voltage (see Fig. 6(b)). By combining a voltage bias with a voltage difference across the electrodes, variable rotations and deflections can be achieved (see Fig. 6(c)). The electrostatic-structural coupled-field solver (ESSOLV) in ANSYS was used to predict displacement due to uniform voltages applied to both electrodes (See Fig. 8(a)).

Modal analysis demonstrated the capability of this mirror design to achieve a desired mode of pure bending of the beam and also a mode with the mirror plate rotating about the beam axis (See Table 2). The mirror can resonate in these modes with a lower voltage than needed for static deflection which is advantageous for optical switching applications. 


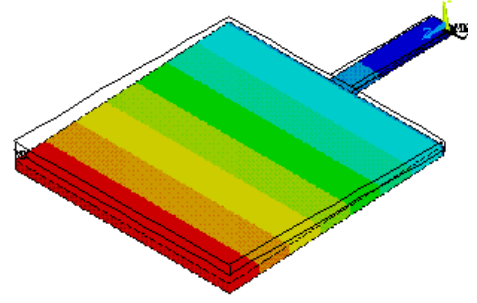

(a)

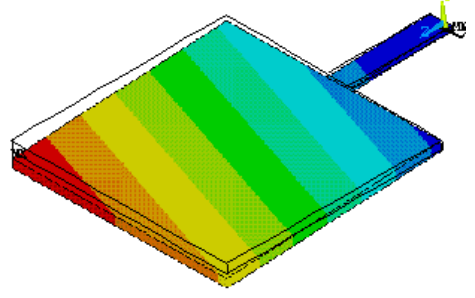

(b)

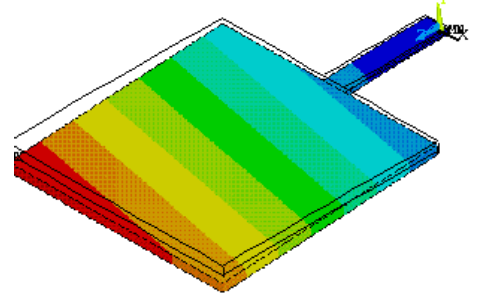

(c)

Figure 6. FEA electrostatic-structural displacement results for cantilevered beam mirror. a.) Bias voltage of 8 Volts applied to both electrodes. b.) Voltage of 8 Volts applied only to left electrode. c.) Bias voltage applied to both electrodes and 8 Volts also applied to left electrode.

Table 2. First three modes of cantilevered beam mirror design as modeled in FEA software.

\begin{tabular}{|c|c|c|}
\hline Mode 1 & Mode 2 & Mode 3 \\
\hline $30.749 \mathrm{kHz}$ & $110.184 \mathrm{kHz}$ & $143.065 \mathrm{kHz}$ \\
beam bending & rotation about beam axis & in-plane twisting \\
(See Fig. 7(a)) & (See Fig. 7(b)) & (See Fig. 7(c)) \\
\hline
\end{tabular}

\subsection{Analytical Model of Cantilevered Beam Mirror}

The static behavior of the cantilevered beam mirror design has been modeled using the equations of slope angle and deflection as a function of applied moment for an anchored cantilevered beam ${ }^{7}$ where

$$
\begin{gathered}
\theta_{A}=\frac{M_{o} l}{E I}, \\
y_{A}=\frac{-M_{o} l^{2}}{2 E I}=\frac{-\theta_{A} l}{2} .
\end{gathered}
$$

$\theta_{A}$ is the slope angle of the beam deflection and $y_{A}$ is the vertical distance which the end of the beam has been moved from it's initial horizontal position, $l$ is the length of the beam, $E$ is Young's Modulus of Polysilicon, and $I$ is the moment of inertia of the beam cross-section. For the case of the mirror plate attached to the end of a cantilevered beam the total displacement can be calculated by (See Fig.5(b))

$$
\left|y_{\text {total }}\right|=\left|y_{A}\right|+\left|y_{m}\right|=\frac{\theta_{A} l}{2}+L \theta_{A}
$$

To find the static relationship between applied voltage and angular displacement of the mirror and beam, the calculation of electrostatic torque acting on the mirror was modeled after the analysis presented by Toshiyoshi and Fujita. ${ }^{2} \quad$ After integration of the electrostatic pressure over the length of the mirror it follows that the total electrostatic torque acting on the mirror is

$$
T_{e}=\frac{\varepsilon \beta V^{2} w}{2 \theta^{2}}, \beta=\left\{\frac{L \sin \theta}{\left(d-\left|y_{A}\right|\right)-L \sin \theta}+\ln \left(1-\frac{L \sin \theta}{d-\left|y_{A}\right|}\right)\right\}
$$

where $\varepsilon$ is the free space permittivity of air, $V$ is the voltage applied to both electrodes, $w$ is the combined width of the electrodes, $\theta$ is $\theta_{A}$ from Equation (5), $L$ is the length of the mirror, and $\mathrm{d}$ is the length of the gap between the mirror under surface and the electrodes. Substituting Equation (8) into Equation (5) a relationship between applied voltage and the beam bending angle is obtained as

$$
V^{2}=\frac{2 \theta^{3} E I}{l \varepsilon w \beta}
$$




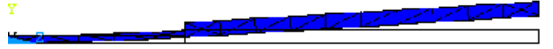

(a)



(b)

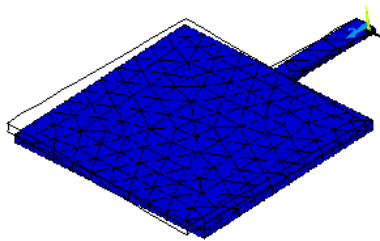

(c)

Figure 7. a.) Mode 1. Side view of mirror and beam bending b.) Mode 2. Front view of mirror rotating about beam axis. c.) Mode 3. Beam and mirror shifting in-plane .

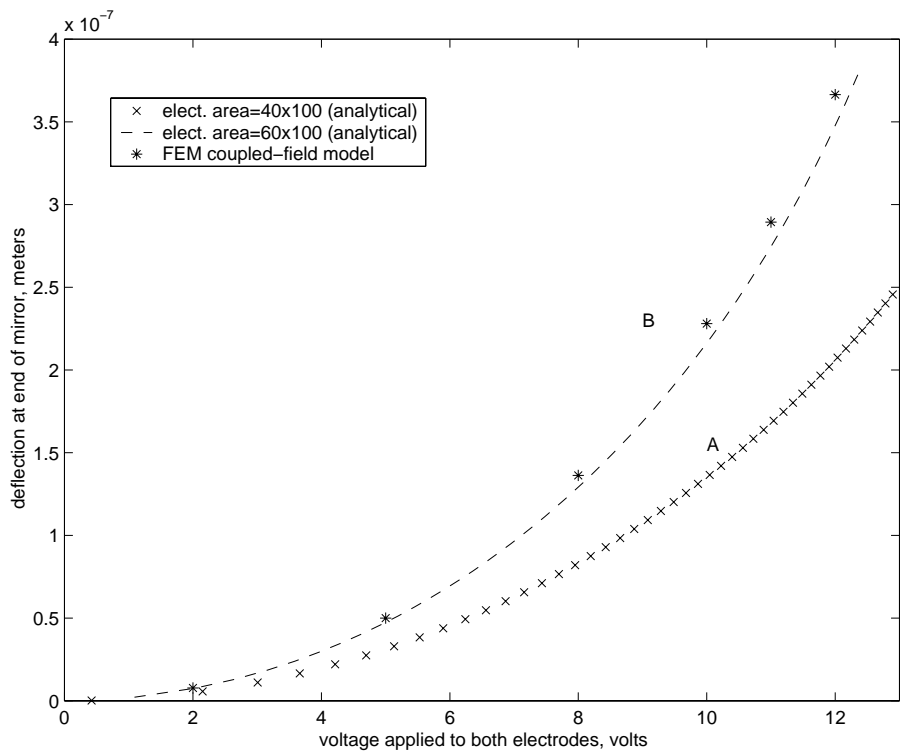

(a)

Figure 8. a.) Deflection at end of mirror vs. voltage applied to both electrodes calculated by analytical model and ANSYS coupled-field model. Curve A is calculated using the actual area of the electrodes under the mirror (40x100 $\left.\mu \mathrm{m}^{2}\right)$. Curve B is calculated using an increased area $\left(60 \times 100 \mu \mathrm{m}^{2}\right)$ to account for fringing-field effects and correlates well with FEM coupled-field model results.

and the maximum vertical deflection is calculated by Eq.(7). This relationship is plotted with the results from the coupled-field model to show the maximum displacement occurring at the end of the mirror versus the applied voltage to both electrodes (See Fig. 8(a)). When the actual width of the electrodes (20 $\mu$ m per electrode) is used in Equation (9), the displacement is underestimated as compared with the FEA model results which is explained by assuming negligent fringing field effects in Equation (8). By increasing the total combined width of the electrodes to $60 \mu \mathrm{m}$ in the analytical model to account for the extra torque added by the fringing fields, the results correlate closely with the coupled-field FEM model.

\section{CONCLUSIONS}

Micromirrors for large optical switching arrays will need to be controllable to precise positions using 2 or 3-DOF motion and advanced control architectures. Our analysis of two different designs of a 2-DOF micromirror shows that electrostatic interference in a gimbal design with four electrodes inhibits independent rotations of the mirror and frame and would therefore be difficult to control to precise positions. The new design proposed in this paper offers desirable modes for optical switching applications and exhibits less electrostatic interference than the gimbal 
design. With only two electrodes, compact arrays can be arranged because less on-chip wiring will be required for addressing individual electrodes. A prototype of both mirror designs have been fabricated by surface micromachining technology to verify results experimentally.

\section{REFERENCES}

1. S.-S. Lee and L.-S. Huang, "2x2 mems fiber optic switches with silicon submount for low-cost packaging," SolidState Sensor and Actuator Workshop, Hilton Head Island, South Carolina, pp. 281-284, 1998.

2. H. Toshiyoshi and H. Fujita, "Electrostatic micro torsion mirrors for an optical switch matrix," Journal of Microelectromechanical Systems 5(4), pp. 231-237, 1996.

3. V. A. Aksyuk and B. P. Barber, Micro-Opto-Electromechanical Devices and Method Therfor, Patent Number US-5995688, United States Patent Office, 1999.

4. V. Aksyuk and F. Pardo, "Lucent microstar micromirror array technology for large optical crossconnects," in MOEMS and Miniaturized Systems, M. E. Motamedi and R. G eds.

5. A. Gasparyan and V. Aksyuk, "Mechanical reliability of surface-micromachined self-assembling two-axis mems tilting mirrors," in MEMS Reliability for Critical Applications, R. A. Lawton, ed., Proceedings of SPIE 4180, pp. 86-90, 2000.

6. J. I. Young and A. M. Shkel, Micromachined 2-DOF Micromirror for Precision Light Manipulation, University of California Irvine Office of Technolgy Alliances, Disclosure Application Date: February 10, 2001.

7. W. C. Young, Roark's Formulas for Stress and Strain, McGraw Hill Inc., New York, 1989. 